\title{
Total metabolic tumor volume as a survival predictor for patients with diffuse large B-cell lymphoma in the GOYA study
}

Lale Kostakoglu, ${ }^{1}$ Federico Mattiello, ${ }^{2}$ Maurizio Martelli, ${ }^{3}$ Laurie H. Sehn, ${ }^{4}$ David Belada, ${ }^{5}$ Chiara Ghiggi, ${ }^{6}$ Neil Chua, ${ }^{7}$ Eva González-Barca,${ }^{8}$ Xiaonan Hong, ${ }^{9}$ Antonio Pinto, ${ }^{10}$ Yuankai Shi, ${ }^{11}$ Yoichi Tatsumi, ${ }^{12}$ Christopher Bolen, ${ }^{13}$ Andrea Knapp, ${ }^{2}$ Gila Sellam, ${ }^{2}$ Tina Nielsen, ${ }^{2}$ Deniz Sahin, ${ }^{2}$ Umberto Vitolo ${ }^{14}$ and Marek Trněný ${ }^{15}$

'Department of Radiology and Medical Imaging, University of Virginia, Charlottesville, VA, USA; ${ }^{2} \mathrm{~F}$. Hoffmann-La Roche Ltd, Basel, Switzerland; ${ }^{3}$ Department of Translational and Precision Medicine, Sapienza University, Rome, Italy; ${ }^{4} \mathrm{BC}$ Cancer Center for Lymphoid Cancer and the University of British Columbia, Vancouver, British Columbia, Canada; ${ }^{5}$ Fourth Department of Internal Medicine-Hematology, Charles University, Hospital and Faculty of Medicine, Hradec Králové, Czech Republic; ${ }^{6}$ Universitaria San Martino, Genoa, Italy; ${ }^{7}$ Cross Cancer Institute, University of Alberta, Edmonton, Alberta, Canada; ${ }^{8}$ Institut Català d'Oncologia, Institut d'Investigació Biomédica de Bellvitge, Universitat de Barcelona, Hospitalet de Llobregat, Barcelona, Spain; ${ }^{9} \mathrm{Fudan}$ University Shanghai Cancer Center, Shanghai, China; ${ }^{10} \mathrm{Hematology-Oncology,} \mathrm{Istituto} \mathrm{Nazionale} \mathrm{Tumori,} \mathrm{Fondazione} \mathrm{G.} \mathrm{Pascale,}$ IRCCS, Naples, Italy; ${ }^{11}$ Department of Medical Oncology, Beijing Key Laboratory of Clinical Study on Anticancer Molecular Targeted Drugs, National Cancer Center/Cancer Hospital, Chinese Academy of Medical Sciences and Peking Union Medical College, Beijing, China; ${ }^{12}$ Department of Patient Safety and Management, Kindai University Hospital and Department of Hematology and Rheumatology, Kindai University Faculty of Medicine, Osaka, Japan; ${ }^{13}$ Genentech, Inc., South San Francisco, CA, USA; ${ }^{14}$ Multidisciplinary Oncology Outpatient Clinic, Candiolo Cancer Institute, FPO-IRCCS, Candiolo, Turin, Italy and ${ }^{15}$ First Department of Medicine, Charles University General Hospital, Prague, Czech Republic.

\author{
Correspondence: \\ Lale Kostakoglu \\ lk3qf@virginia.edu \\ Received: $\quad$ March 1, 2021. \\ Accepted: $\quad$ August 4, 2021. \\ Prepublished: $\quad$ August 19, 2021. \\ https://doi.org/10.3324/haematol.2021.278663 \\ ๑2022 Ferrata Storti Foundation \\ Haematologica material is published under a CC \\ BY-NC license @ (ब) $\Theta$
}

\section{Abstract}

This retrospective analysis of the phase III GOYA study investigated the prognostic value of baseline metabolic tumor volume parameters and maximum standardized uptake values for overall and progression-free survival (PFS) in treatment-naïve diffuse large B-cell lymphoma. Baseline total metabolic tumor volume (determined for tumors $>1 \mathrm{~mL}$ using a threshold of 1.5 times the mean liver standardized uptake value +2 standard deviations), total lesion glycolysis, and maximum standardized uptake value positron emission tomography data were dichotomized based on receiver operating characteristic analysis and divided into quartiles by baseline population distribution. Of 1,418 enrolled patients, 1,305 had a baseline positron emission tomography scan with detectable lesions. Optimal cut-offs were $366 \mathrm{~cm}^{3}$ for total metabolic tumor volume and 3,004 g for total lesion glycolysis. High total metabolic tumor volume and total lesion glycolysis predicted poorer PFS, with associations retained after adjustment for baseline and disease characteristics (high total metabolic tumor volume hazard ratio: 1.71, 95\% confidence interval [CI]: 1.352.18; total lesion glycolysis hazard ratio: 1.46; 95\% Cl: 1.15-1.86). Total metabolic tumor volume was prognostic for PFS in subgroups with International Prognostic Index scores 0-2 and 3-5, and those with different cell-of-origin subtypes. Maximum standardized uptake value had no prognostic value in this setting. High total metabolic tumor volume associated with high International Prognostic Index or non-germinal center B-cell classification identified the highest-risk cohort for unfavorable prognosis. In conclusion, baseline total metabolic tumor volume and total lesion glycolysis are independent predictors of PFS in patients with diffuse large B-cell lymphoma after first-line immunochemotherapy.

\section{Introduction}

Current standard of care for patients with previously untreated diffuse large B-cell lymphoma (DLBCL) is immunochemotherapy with the anti-CD20 antibody rituximab in combination with cyclophosphamide, doxorubicin, vincristine, and prednisone (CHOP)., ${ }^{1,2}$ DLBCL is a heterogeneous disease that is reflected in transcriptionally defined, molecularly distinct subtypes. . $^{3,4}$ Consequently, $10-15 \%$ of patients have primary refractory disease, and $20-30 \%$ of 
patients experience relapse after initial response to firstline therapy. ${ }^{5}$ Identification of high-risk patients is challenging with the currently available risk-stratification models. ${ }^{6,7}$ Gene expression profiling and other molecular signatures can predict outcome, independently of clinical International Prognostic Index (IPI) score. ${ }^{3,4}$

${ }^{18} \mathrm{~F}$-fluorodeoxyglucose (FDG) positron emission tomography (PET)/computed tomography (CT) is an important tool for the diagnosis, staging, and early evaluation of response to therapy in patients with DLBCL. FDG-PET/CT together with baseline quantitative measures of FDG uptake, which act as indicators of metabolically active whole-body disease burden, have demonstrated good prognostic value for the prediction of long-term survival outcomes in a number of small studies of patients with previously untreated DLBCL. ${ }^{8-15}$ Examples of quantitative PET variables currently being explored for potential prognostic value include total metabolic tumor volume (TMTV), total lesion glycolysis (TLG), and maximum standardized uptake value $\left(S \cup V_{\max }\right)$. Other studies have investigated the prognostic value of interim response assessment by PET, with this having limited predictive value, ${ }^{16,17}$ or end-oftreatment complete response by PET, which was shown to be associated with improved survival outcomes. ${ }^{18-20}$ The phase III GOYA study (clinicaltrials gov. Identifier: NCT01287741) compared the glycoengineered type II antiCD20 antibody, obinutuzumab, plus CHOP with rituximab plus CHOP in patients with previously untreated DLBCL. ${ }^{2}$ Although improvements in progression-free survival (PFS) have been demonstrated with obinutuzumab-versus rituximab-based therapy in follicular lymphoma ${ }^{22}$ and chronic lymphocytic leukemia, ${ }^{23}$ no significant PFS difference was found between the obinutuzumab and rituximab arms in the GOYA study (hazard ratio [HR]: 0.92; 95\% confidence interval $[\mathrm{Cl}]$ : 0.76-1.11; $P=0.39) .{ }^{21}$ Here, we report a post hoc analysis of data from the GOYA study, evaluating the prognostic value of baseline quantitative PET parameters (TMTV, TLG and SUV ${ }_{\max }$ ), independently and combined with other risk factors, as potential prognostic markers for PFS and overall survival (OS) in this large population of patients with DLBCL. In GOYA, no statistically significant difference in PFS was found between treatment arms ${ }^{21}$ so the two arms were pooled for this post hoc analysis.

\section{Methods}

GOYA was a phase III, open-label, multicenter, randomized study. The study design and patient disposition have been described previously (see the Online Supplementary Appendix). ${ }^{21}$ GOYA was conducted in accordance with the International Conference on Harmonisation guidelines for Good Clinical practice, and the protocol was approved by the ethics committees of participating centers. Patients provided written informed consent.

FDG-PET/CT scans were performed at baseline ( $\leq 35$ days before the first dose of study treatment) and at end of treatment (6-8 weeks after the last antibody treatment), or in the event of early discontinuation (4-8 weeks after the last dose of antibody treatment). TMTV, TLG, and SUV $_{\max }$ were centrally determined by three experienced nuclear medicine physicians from each baseline PET/CT scan collected in real time during the study. TMTV was calculated using a tumor threshold of 1.5 times the mean SUV of the liver +2 standard deviations. Only those tumors that measured $>1 \mathrm{~mL}$ were included in the TMTV calculation. Gene expression profiling using the NanoString Lymphoma Subtyping Research-Use-Only assay (NanoString Technologies, Inc., Seattle, WA, USA) was used for cell-oforigin classification.

The primary objective of this retrospective analysis was to determine the prognostic value of baseline quantitative PET parameters (TMTV, TLG, and SUV ${ }_{\text {max }}$ ) for OS and PFS in the overall patient population. Secondary objectives were to investigate their prognostic value according to IPI risk category and DLBCL cell-of-origin subtype.

\section{Statistical analysis}

Statistical analyses were performed on the PET intent-totreat (ITT) population, which included all randomized patients with a baseline PET scan with detectable lesions.

PFS and OS from randomization were estimated using the Kaplan-Meier method. In order to determine the optimal cut-offs, baseline TMTV and TLG were dichotomized based on time-dependent receiver operating characteristic (ROC) curves, selecting the 24-month landmark (i.e., considering investigator-assessed PFS events that occurred between randomization and 2 years). PFS and OS were evaluated according to these cut-offs and also an a priori cut-off of $300 \mathrm{~cm}^{3}$ (not validated) for TMTV. ${ }^{12}$ Covariate effects on PFS were estimated using multivariable Cox models, including treatment and study stratification factors. Hazard ratios were stratified for IPI score and number of planned chemotherapy cycles.

Baseline TMTV values were dichotomized according to the ROC-derived cut-off, while for TLG, data were dichotomized according to the median TLG value at baseline. Quartiles of TMTV and TLG were defined based on their distribution in the available population at baseline. Associations between PFS and OS, and baseline TMTV and TLG quartiles, were assessed using the Kaplan-Meier method. The association between PFS and baseline TMTV and TLG quartiles was also evaluated separately in patients with germinal center $B$ cell (GCB) and activated B cell (ABC) cell-of-origin subtypes, and in patients with IPI scores of 0-2 and 3-5. Impact of PET parameters on PFS was also evaluated, adjusting for IPI score as well as cell-of-origin subtype, to determine the prognostic value they provide 
in addition to other known factors.

Statistical analyses were performed using SAS v9.4 (SAS Institute, Inc., Cary, NC, USA); $P$ values $<0.05$ were considered significant.

\section{Results}

\section{Patient population and baseline demographics}

The GOYA study enrolled 1,418 patients between July 2011 and June 2014, with 1,414 patients included in the final analysis population (GOYA ITT population). Of these, 1,305 (92.3\%) had a PET scan with detectable lesions at baseline from which quantitative data could be obtained (PET ITT population; see the Online Supplementary Appendix). Baseline demographics and disease characteristics according to dichotomized baseline TMTV values in the PET ITT population are displayed in Table 1. Demographics and baseline characteristics were similar in the GOYA ITT and PET ITT populations (Online Supplementary Table S1). The proportion of patients with an IPI score of 3-5, Ann Arbor stage III/IV, Eastern Cooperative Oncology Group performance status (ECOG PS) $\geq 2$, extranodal involvement and elevated serum lactate dehydrogenase (LDH) was significantly greater in the high versus low TMTV group, and the high
TMTV group also had a significantly greater median sum of products of diameter (SPD) of up to six target lesions (Table 1). TMTV was associated with median time to randomization, with a shorter median time in the high versus low TMTV group (0.69 vs. 0.92 months). Dichotomized TLG data and both TMTV and TLG quartiles showed a similar overall pattern to dichotomized TMTV data, with respect to the two study populations, clinical characteristics and median time to randomization (Online Supplementary Tables S2 and S3). There was no association between quantitative PET measures and cell-of-origin.

Based on the data cut-off of January 31, 2018 for the final analysis of GOYA, the median duration of follow-up from randomization to last contact or death was 48 months (range, 0.1-78 months).

\section{Association between baseline quantitative positron emission tomography parameters and investigator-assessed progression-free and overall survival}

Total metabolic tumor volume

ROC analysis identified $366 \mathrm{~cm}^{3}$ as the optimal cut-off for determining the association between TMTV and PFS. Using this cut-off, Kaplan-Meier analysis showed that PFS was shorter for patients with high versus low TMTV

Table 1. Demographics and baseline characteristics according to high $\left(\geq 366 \mathrm{~cm}^{3}\right)$ or low $\left(<366 \mathrm{~cm}^{3}\right)$ baseline total metabolic tumor volume in the positron emission tomography intent-to-treat population $(n=1,305)$.

\begin{tabular}{|c|c|c|c|}
\hline & $\begin{array}{l}\text { High TMTV } \\
(\mathrm{N}=636)\end{array}$ & $\begin{array}{l}\text { Low TMTV } \\
(\mathrm{N}=669)\end{array}$ & $P *$ \\
\hline Median age, years (range) & $61.0(18-86)$ & $62.0(18-85)$ & 0.003 \\
\hline Male, N (\%) & $358(56.3)$ & $333(49.8)$ & 0.0198 \\
\hline $\begin{array}{l}\text { Median time from diagnosis } \\
\text { to randomization, months (range) }\end{array}$ & $\begin{array}{c}{[N=632]} \\
0.69(0.1-36.3)\end{array}$ & $\begin{array}{c}{[N=668]} \\
0.92(0.0-8.7)\end{array}$ & 0 \\
\hline $\begin{array}{l}\text { Ann Arbor stage, N (\%) } \\
\text { I or II } \\
\text { III or IV }\end{array}$ & $\begin{array}{c}91(14.3) \\
545(85.7)\end{array}$ & $\begin{array}{l}223(33.3) \\
446(66.7)\end{array}$ & 0 \\
\hline $\begin{array}{l}\text { IPI score, N (\%) } \\
0-2 \\
3 \\
4-5\end{array}$ & $\begin{array}{l}251(39.5) \\
234(36.8) \\
151(23.7)\end{array}$ & $\begin{array}{c}484(72.3) \\
140(20.9) \\
45(6.7)\end{array}$ & 0 \\
\hline $\begin{array}{l}\text { ECOG PS, N (\%) } \\
\begin{array}{l}0-1 \\
2-3\end{array}\end{array}$ & $\begin{array}{l}{[\mathrm{N}=635]} \\
521(82.0) \\
114(18.0)\end{array}$ & $\begin{array}{c}- \\
623(93.1) \\
46(6.9)\end{array}$ & 0 \\
\hline Any extranodal involvement, N (\%) & $458(72.0)$ & $418(62.5)$ & 0.0003 \\
\hline Serum LDH elevated, N (\%) & {$[N=633] 501(79.1)$} & {$[\mathrm{N}=668] 244(36.5)$} & 0 \\
\hline $\begin{array}{l}\text { Median SPD, mm } \\
\text { (range) }\end{array}$ & $\begin{array}{c}8,198 \\
(160-510,000)\end{array}$ & $\begin{array}{c}{[\mathrm{N}=666] 2,570} \\
(0-194,400)\end{array}$ & 0 \\
\hline $\begin{array}{l}\text { Cell-of-origin subtype, N (\%) } \\
\text { GCB } \\
\text { ABC } \\
\text { Unclassified }\end{array}$ & $\begin{array}{l}{[N=410]} \\
239(58.3) \\
103(25.1) \\
68(16.6)\end{array}$ & $\begin{array}{l}{[N=451]} \\
255(56.5) \\
124(27.5) \\
72(16.0)\end{array}$ & 0.740 \\
\hline
\end{tabular}

*Kruskal-Wallis rank sum test (numeric variables) or Fisher's exact test (categorical variables). ABC: activated B-cell-like; ECOG PS: Eastern Cooperative Oncology Group performance status; GCB: germinal center B-cell-like; IPI: International Prognostic Index; LDH: lactate dehydrogenase; SPD: sum of products of diameter of up to six target lesions; TMTV: total metabolic tumor volume. 
A

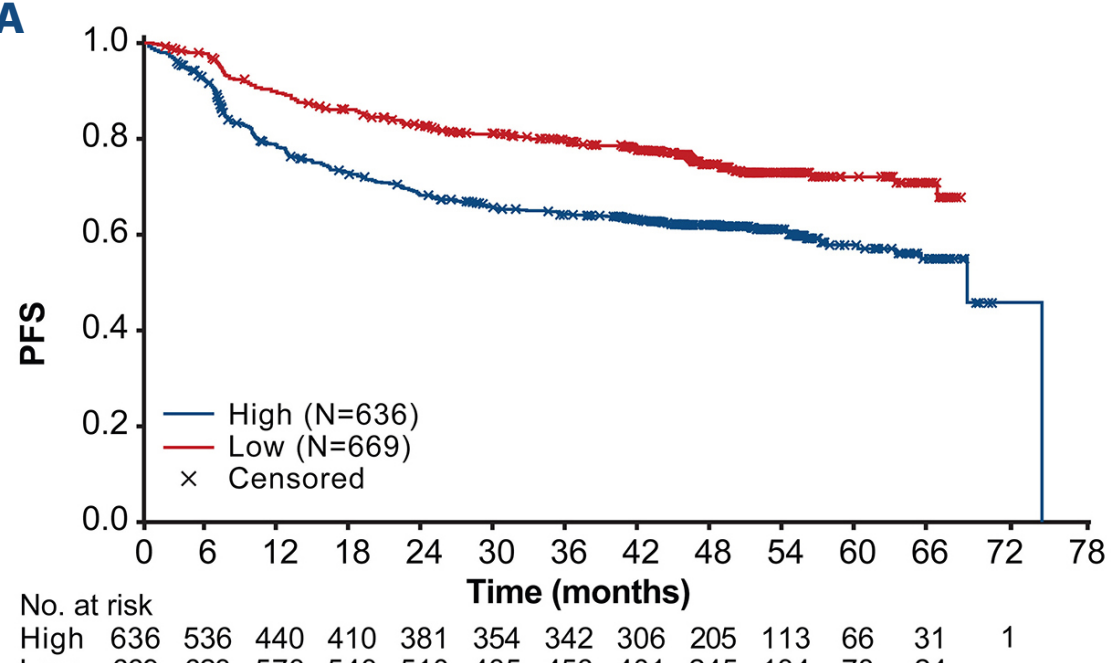

B

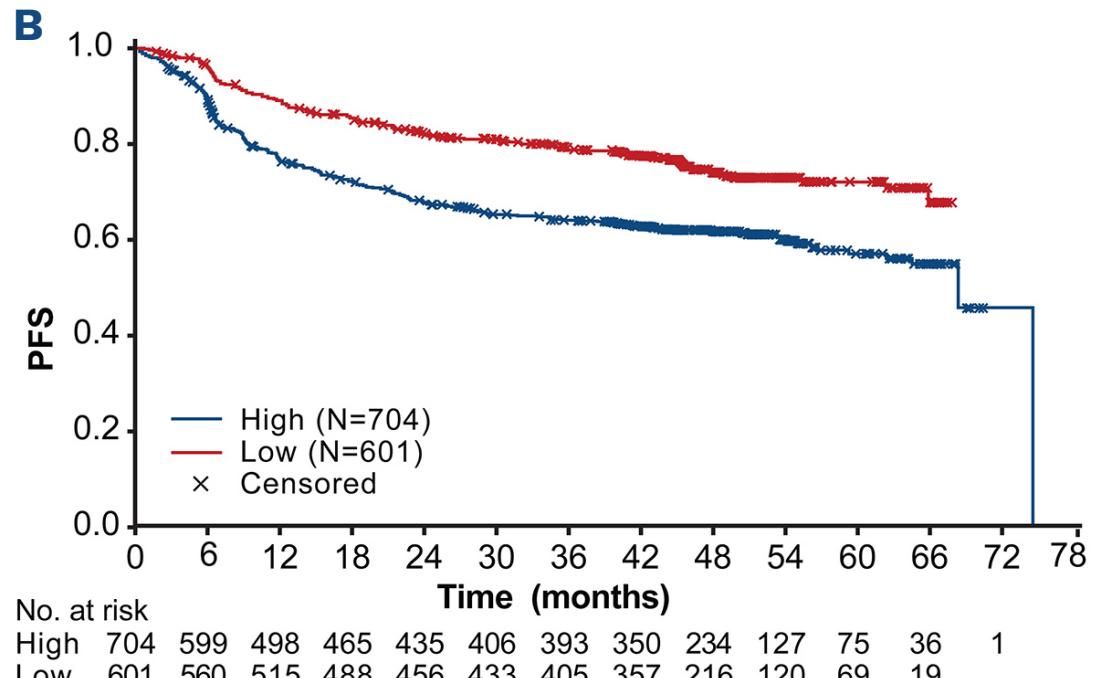

Figure 1. Kaplan-Meier analysis of investigator-assessed progression-free survival according to baseline total metabolic tumor volume cut-offs. Total metabolic tumor volume (TMTV) cut-off of (A) $366 \mathrm{~cm}^{3}$ and (B) $300 \mathrm{~cm}^{3}$.

$\begin{array}{lcc}\text { Covariate } & \text { Hazard ratio }(95 \% \text { CI) } & \boldsymbol{P} \\ \text { TMTV, high vs. low } & 1.95(1.59-2.38) & 0 \\ \text { TLG, high vs. low } & 1.71(1.40-2.09) & <0.0001 \\ \text { Treatment group, obinutuzumab vs. rituximab } & 0.95(0.78-1.16) & 0.627 \\ \text { Sex, female vs. male } & 0.95(0.78-1.15) & 0.577 \\ \text { Age at randomization } & 1.01(1.00-1.02) & 0.032 \\ \text { Time from diagnosis to randomization } & 0.79(0.67-0.94) & 0.009 \\ \text { Geographic region } & & \\ \text { Eastern Europe vs. Asia } & 0.71(0.51-0.99) & 0.043 \\ \text { Western Europe vs. Asia } & 0.69(0.55-0.88) & 0.002 \\ \text { North America vs. Asia } & 0.73(0.54-0.98) & 0.035 \\ \text { Other vs. Asia } & 0.75(0.45-1.26) & 0.279 \\ \text { Ann Arbor stage } & & \\ \text { II vs.I } & 1.13(0.66-1.94) & 0.660 \\ \text { III vs.I } & 1.65(1.00-2.70) & 0.048 \\ \text { IV vs.I } & 2.24(1.39-3.63) & 0.001 \\ \text { ECOG PS, 2-3 vs. 0-1 } & 1.71(1.32-2.23) & <0.0001 \\ \text { Extranodal involvement, yes vs. no } & 1.32(1.06-1.63) & 0.013 \\ \text { Serum LDH, elevated vs. not elevated } & 1.37(1.12-1.68) & 0.002 \\ \text { SPD } & 1.00(1.00-1.00) & 0.318 \\ \text { IPI, 3-5 vs. 0-2 } & 1.69(1.39-2.05) & <0.0001\end{array}$

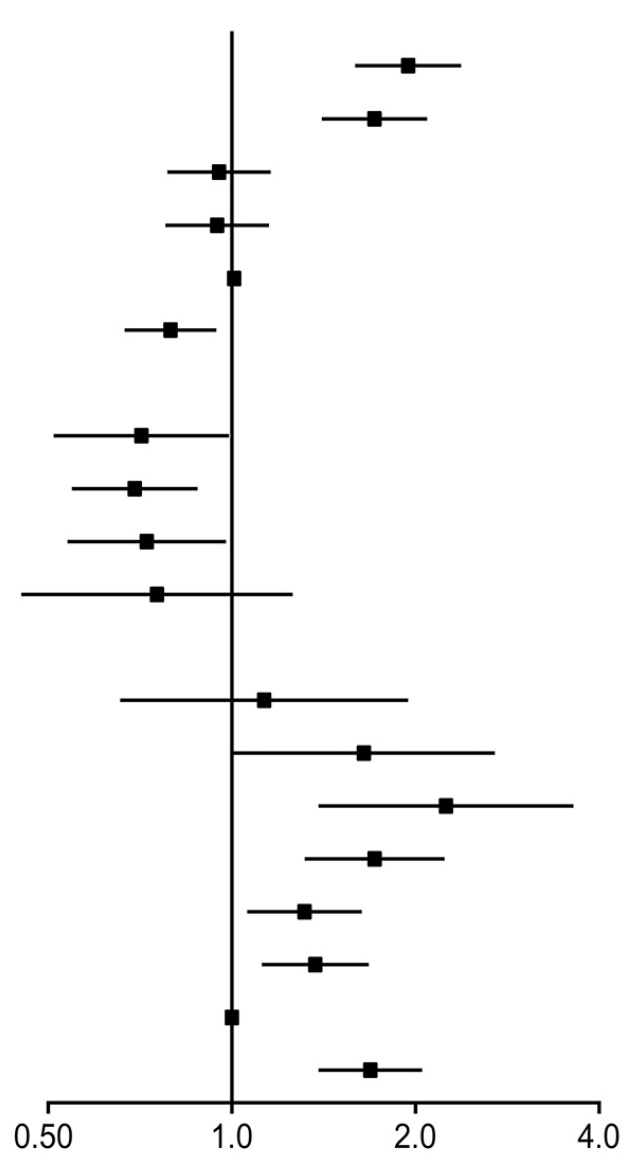

Figure 2. Univariate Cox regression model evaluating factors associated with investigator-assessed progression-free survival (positron emission tomography intent-to-treat population; $\mathbf{n}=\mathbf{1 , 3 0 5}$ ). Total metabolic tumor volume (TMTV) high defined as $\geq 366 \mathrm{~cm}^{3}$ and TMTV low defined as $<366 \mathrm{~cm}^{3}$; TLG high defined as $\geq$ median total lesion glycolysis (TLG) and TLG low defined as <median TLG. CI: confidence interval; ECOG PS: Eastern Cooperative Oncology Group performance status; IPI: International Prognostic Index; LDH: lactate dehydrogenase; SPD: sum of products of diameters of up to 6 target lesions.

(Figure $1 \mathrm{~A})$, with a stratified HR for PFS of $1.73(95 \% \mathrm{Cl}$ : 1.40-2.13) $(P<0.0001)$. The same association was identified when the a priori cut-off of $300 \mathrm{~cm}^{3}$ was evaluated (stratified HR: 1.53; 95\% Cl: 1.24-1.90; P<0.0001; Figure 1B). Four-year PFS rates were lower for patients with baseline TMTV $\geq 366 \mathrm{~cm}^{3}$ compared with $<366 \mathrm{~cm}^{3}$ (4-year rate: $59.7 \%$ vs. $74.7 \%$ ), and univariate followed by multivariable Cox regression analysis confirmed the association between high TMTV and shorter PFS (multivariable analysis: HR: 1.71; 95\% Cl: 1.35-2.18; $P<0.0001$; Figure 2; Online Supplementary Figure S1A). Inferior PFS was also independently associated with other characteristics in the multivariable model, including Asian geographic region (Western European vs. Asian region: HR: 0.61; 95\% Cl: 0.48-0.78; $P<0.0001 ;$ North American vs. Asian region: hazard ratio: $0.63 ; 95 \% \mathrm{Cl}: 0.47-0.86 ; P=0.004)$. The association between high TMTV and poorer PFS was stronger for the subgroup of patients with an IPI risk score of $3-5$ (HR: $1.93 ; 95 \% \mathrm{Cl}: 1.41-2.65)$ than for those with a score of 0-2 (HR: 1.59; 95\% Cl: 1.18-2.14), when adjusted for treatment group, geographic region, sex and SPD ( $P=0.002$ for the TMTV HR adjusted for interaction with IPI score; Online Supplementary Table S4).

When TMTV was divided into quartiles according to base- 


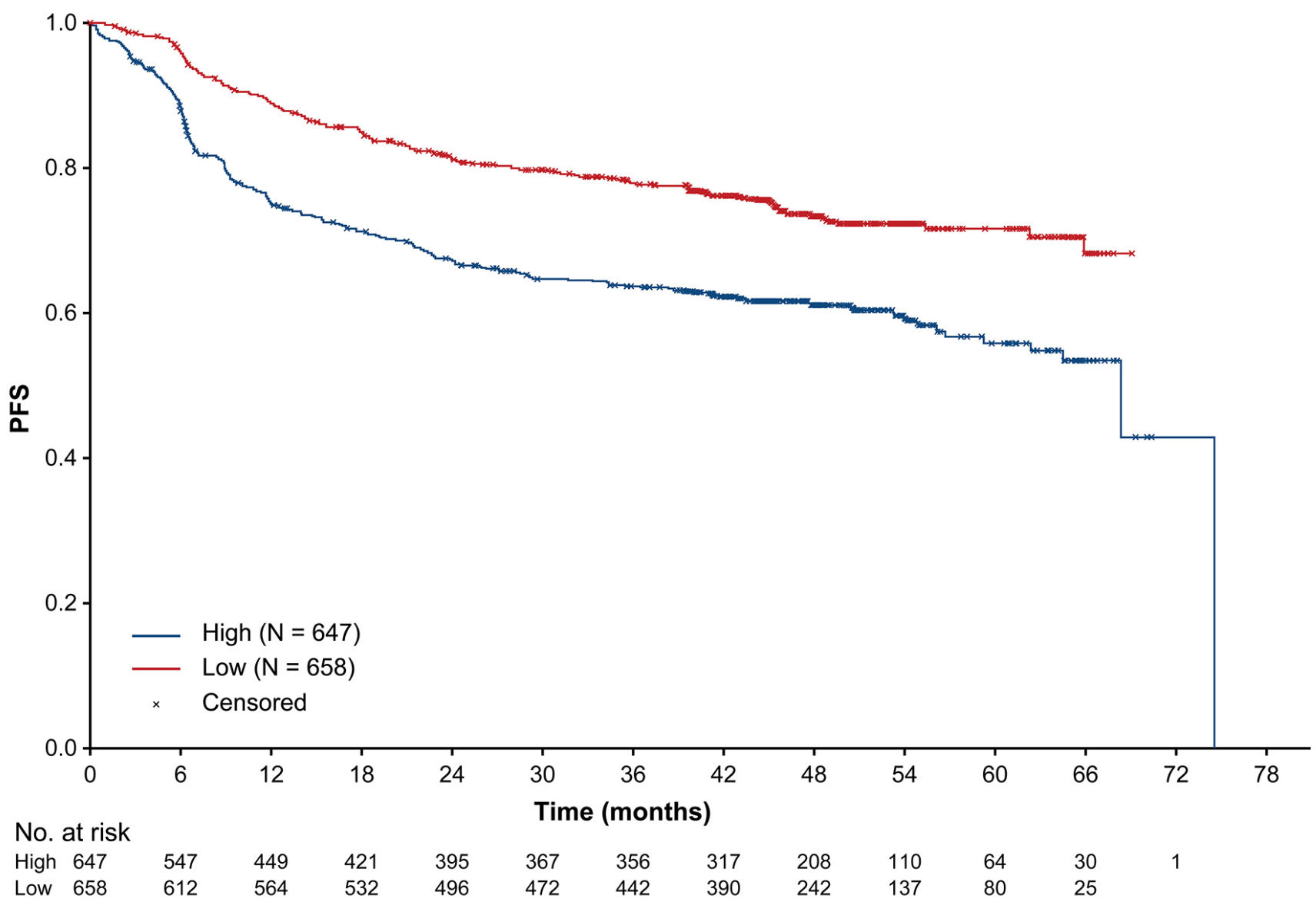

Figure 3. Kaplan-Meier analysis of investigator-assessed progression-free survival according to a baseline total lesion glycolysis cut-off of $\mathbf{3 , 0 0 4}$ g. PFS: progression-free survival; TLG: total lesion glycolysis.

line values, the highest quartile was associated with worse prognosis for investigator-assessed PFS, compared with the lowest quartile in both IPI categories $0-2$ and 3-5 (quartile 4 vs. quartile 1, stratified HR: 1.95; 95\% Cl: 1.452.61; P<0.0001; Online Supplementary Figure S2). KaplanMeier graphs for PFS according to baseline TMTV quartiles in patients with low and high IPI scores are shown in the Online Supplementary Figure S3A and B.

OS was also found to be poorer in patients with high versus low TMTV, based on the tumor volume cut-off of 366 $\mathrm{cm}^{3}$ (stratified HR: 1.76; 95\% Cl: 1.34-2.30; $P<0.0001$; 4-year OS rate: $74.5 \%$ vs. $86.6 \%$ ) and the $300 \mathrm{~cm}^{3}$ cut-off (Online Supplementary Figure S4).

\section{Total lesion glycolysis}

ROC analysis identified a cut-off of $3,004 \mathrm{~g}$ as the optimal value for assessing the association with PFS. Using this cut-off, Kaplan-Meier analysis showed that patients with high TLG had significantly poorer PFS compared with patients who had low TLG $(P<0.0001)$ (Figure 3), with 4-year PFS rates of $61.3 \%$ and $73.5 \%$, respectively. Univariate followed by multivariable Cox regression analysis confirmed the independent association with high TLG (multivariable analysis: HR: 1.46 ; 95\% Cl: 1.15-1.86; $P=0.002$ ) and other characteristics in the model, including Asian geographic region (Western European vs. Asian region: HR: 0.62; 95\% Cl: 0.48-0.79; $P=0.0001 ;$ North American vs. Asian region: HR: $0.64 ; 95 \% \mathrm{Cl}: 0.47-0.88 ; P=0.005$ ) (Figure 2; Online Supplementary Figure S1B). This association was stronger in the subgroup of patients with an IPI risk score of 3-5
(HR: 1.64; 95\% Cl: 1.21-2.23), compared with those who had a score of 0-2 (HR: 1.44; 95\% Cl: 1.07-1.94), when adjusted for treatment group, geographic region, sex and SPD $(P=0.016$ for the TLG hazard ratio adjusted for interaction with IPI score) (Online Supplementary Table S4).

When TLG was divided into quartiles according to baseline values, the highest quartile was associated with worse prognosis for investigator-assessed PFS, compared with the lowest quartile (quartile 4 vs. quartile 1, stratified HR: 1.73; 95\% Cl: 1.30-2.31; $P<0.0001)$. Kaplan-Meier graphs for PFS according to baseline TLG quartiles in patients with low and high IPI scores are shown in Online Supplementary Figure S3C and D.

Maximum standardized uptake value

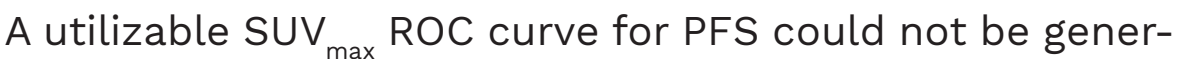
ated, and it was therefore impossible to define a reasonable cut-off for this parameter. An alternative ROC curve, generated for 2-year PFS, was also not prognostic for survival (Online Supplementary Figure S5).

\section{Prognostic value of baseline quantitative positron emission tomography parameters according to cell-of-origin subtype}

DLBCL cell-of-origin subtype information was available for 861 patients in the PET-evaluable population (GCB, $n=494$ [57.4\%]; $A B C, n=227$ [26.4\%]; unclassified, $n=140$ [16.3\%]). The baseline characteristics for patients with available cell-of-origin subtype information (data not shown) were similar to those for the overall PET ITT population. 


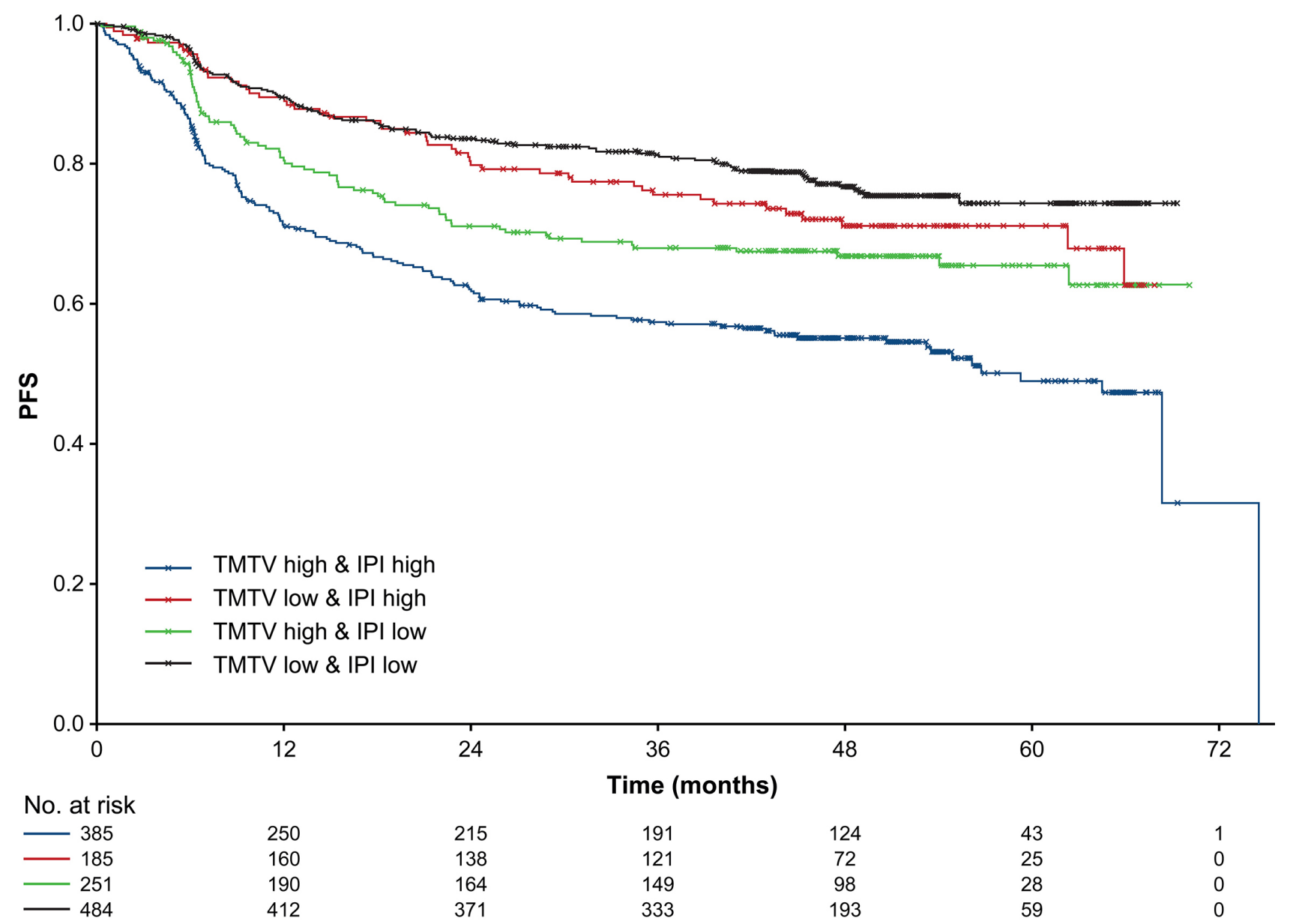

Figure 4. Kaplan-Meier analysis of investigator-assessed progression-free survival according to baseline International Prognostic Index status and a total metabolic tumor volume cut-off of $\mathbf{3 6 6} \mathbf{~ c m}^{3}$. Differences in progression-free survival (PFS) probability were significant between each subgroup $(P<0.0001)$ : IPI: International Prognostic Index; TMTV: total metabolic tumor volume.

High TMTV was prognostic for poorer PFS in patients with both $A B C /$ unclassified DLBCL and in those with GCB DLBCL. Additionally, when assessed according to quartiles, higher TMTV was prognostic for poorer investigator-assessed PFS in patients with $A B C /$ unclassified DLBCL (quartile 4 vs. quartile 1, stratified HR: 2.46; $95 \%$ Cl: $1.49-4.07 ; P=0.0003)$ and in those with GCB DLBCL (stratified HR: 2.51; 95\% Cl: 1.44-4.38; $P=0.0003$ ) (Online Supplementary Figure $S 6 A$ and $B$ ). Similar findings were observed for higher TLG (quartile 4 vs. quartile 1, ABC/unclassified: stratified HR: 2.10; $95 \% \mathrm{Cl}$ : 1.28-3.45; $P=0.0022$; GCB: stratified HR: 2.16 ; $95 \% \mathrm{Cl}: 1.27-3.68$; $P<0.0001$; Online Supplementary Figure S6C, $D$ ).

Similar to findings obtained for the PET ITT population, in the cell-of-origin subtype analysis, high TMTV was prognostic independently of IPI status based on the Cox multivariable analysis (Online Supplementary Table S5). The results for TLG were very similar to those obtained for TMTV (data not shown).

\section{Multivariable analysis with IPI and cell-of-origin classification}

The multivariable analysis with dichotomized TMTV and IPI score or cell-of-origin subtype further stratified the risk groups and identified the highest risk group with the worst prognosis. Kaplan-Meier analysis with dichotomized IPI score (0-2 and 3-5) and TMTV ( $\geq 366$ and $<366$ groups) showed that patients with low IPI score and low TMTV $(n=484)$ had a $74.3 \% 5$-year PFS compared with $49.0 \%$ for patients with high IPI score and high TMTV $(n=385)$; values for patients with high IPI score and low TMTV $(n=185)$ were $71.1 \%$ compared with $65.5 \%$ for low IPI score with high TMTV $(n=251$; Figure 4$)$.

Kaplan-Meier analysis with dichotomized cell-of-origin subtype (GCB vs. non-GCB) and TMTV ( $\geq 366$ and $<366$ groups) showed that patients with GCB subtype and low TMTV $(n=255)$ had an $80.0 \%$ 5-year PFS, compared with $48.8 \%$ for patients with non-GCB subtype and high TMTV $(n=171)$; values for patients with non-GCB subtype and low TMTV $(n=196)$ were $65.1 \%$, compared with $59.7 \%$ for GCB subtype and high TMTV $(n=239)$ (Figure 5$).$

\section{Discussion}

This post hoc analysis of the phase III GOYA study confirms that baseline quantitative PET parameters are prognostic for both PFS and OS in patients with DLBCL receiving first-line immunochemotherapy. Considerably worse prognosis was observed within the highest quartile (quartile 4) compared with the lowest for baseline TMTV (PFS and OS) and TLG (PFS). In general, TLG appeared slightly less prognostic than TMTV, regardless of whether it was split into quartiles or dichotomized. 
ROC analysis identified $366 \mathrm{~cm}^{3}$ as the optimal tumor volume cut-off for TMTV in the GOYA PET ITT population. Despite the ROC cut-off being close to the a priori one and both, in turn, being quite close to the median (366 $\mathrm{cm}^{3}$ represents the $49^{\text {th }}$ percentile), the methodology and the data-driven nature of the $366 \mathrm{~cm}^{3}$ cut-off should be acknowledged, along with the potential for slight overfitting. However, confidence in the prognostic value of the ROC cut-off can be taken from the finding that the stratified HR for PFS and OS were highly similar to those for the a priori cut-off. Thus, a TMTV cut-off of $300-400 \mathrm{~cm}^{3}$ can be suggested for further investigation in future trials with a similar population of DLBCL patients undergoing first-line standard-of-care immunochemotherapy. This cut-off range is similar to values of $300 \mathrm{~cm}^{3}$ and $396 \mathrm{~cm}^{3}$ reported by Cottereau et $a l^{12}$ and Mikhaeel et al.,9 respectively, but somewhat different to values of 220-261 $\mathrm{cm}^{3}$ reported in other studies. ${ }^{13,15,24}$ The variation in reported values is likely due to differences in patient characteristics and the methodology used to measure TMTV. ${ }^{15,25,26}$

The optimal cut-off for TLG was calculated to be $3,004 \mathrm{~g}$, which represented the $49^{\text {th }}$ percentile. This value was within the range of those previously published, ,911-14,24 although there was substantial variation across studies. As for the related parameter of TMTV, differences in pa- tient characteristics and methodology could explain this variation in reported values. ${ }^{15,25,26}$

The current analysis involves the largest DLBCL population to date in which the prognostic value of baseline quantitative PET measures has been assessed and confirmed. Our findings are consistent with other retrospective studies in patients with DLBCL, which have shown high TMTV to be a strong predictor of reduced survival. 9-15,24,27-30 $^{-1}$ Furthermore, a meta-analysis reported a highly significant association between high TMTV and poor PFS (pooled HR: 2.93; 95\% Cl: $2.29-3.73$ ) and OS (pooled HR: 3.52; 95\% Cl: 2.67-4.64) in patients with DLBCL. ${ }^{31}$ Mikhaeel et al. demonstrated that the combination of baseline TMTV and response by PET/CT after two immunochemotherapy cycles (rituximab-CHOP) improved the predictive power of interim PET for PFS, allowing the definition of a poor-prognosis group of DLBCL patients, and was more prognostic than either early response or IPI score in this setting. ${ }^{9}$

For TLG, in agreement with the present analysis, previous retrospective studies have also shown a significant association between high baseline TLG and reduced survival in patients with DLBCL. ${ }^{8,9,24,27,29,32,33}$

Notably, the cut-offs defined for TMTV and TLG in the current study added to the prognostic power of the dichotomized IPI categories ( $0-2$ and $3-5)$. The association between high TMTV and poorer PFS was stronger for the

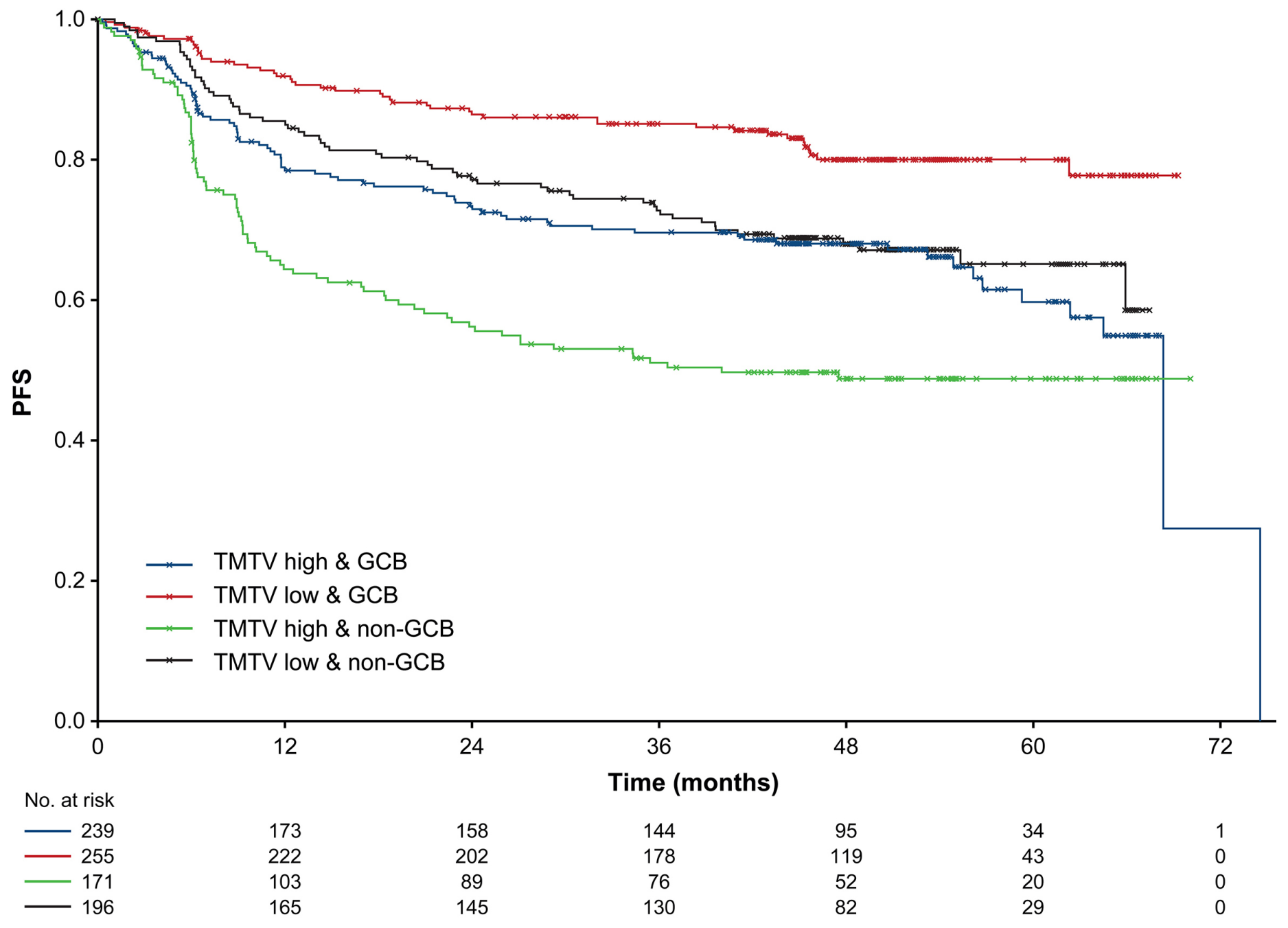

Figure 5. Kaplan-Meier analysis of investigator-assessed progression-free survival according to germinal center B-cell status and a baseline total metabolic tumor volume cut-off of $\mathbf{3 6 6} \mathbf{c m}^{3}$. Differences in progression-free survival (PFS) probability were significant between each subgroup $(P<0.0001)$. GCB: germinal center B-cell-like; TMTV: total metabolic tumor volume. 
subgroup of patients with an IPI risk score of 3-5, than for those with a lower score. This has been previously demonstrated for TMTV, which retained an association with poorer PFS P,11,13,29 $^{9}$ and poorer OS ${ }^{11-13}$ after adjustment for IPI score and other baseline parameters. Furthermore, TMTV provided additional stratification for patients with a high IPI score $(\geq 4)$ into two groups with differing PFS and OS rates at 3 years. ${ }^{24}$ This finding has lent further credence for including TMTV in management decisions in both low and high IPI groups to offer a more individualized treatment approach.

We found that TMTV and TLG were prognostic in both GCB and non-GCB subgroups of DLBCL, suggesting that these quantitative parameters are widely applicable across the different DLBCL subgroups. The Kaplan-Meier analysis with dichotomized TMTV and IPI score or cell-of-origin subtype further stratified risk groups with different outcomes and identified a patient subgroup with the highest risk for treatment failure and unfavorable PFS. Patients with a high TMTV and non-GCB phenotype or high TMTV and a high IPI score (5-year PFS rates of $48.8 \%$ and $49.0 \%$, respectively) were at the highest risk of relapse or progression, compared with those who had a low TMTV and a GCB subtype or low TMTV and low IPI score (5-year PFS rates of $80.0 \%$ and $74.3 \%$, respectively). These findings confirmed similar reports from other retrospective studies performed in smaller numbers of patients. ${ }^{12,13,24}$ This integrated risk modeling could lead to better selection of high-risk patients in whom management should be modified before initiation of first line therapy.

Baseline SUV max $_{\text {mas }}$ wot a suitable parameter for predicting survival outcome in the current study. Previous studies that also used ROC analysis to define an SUV cut-off corroborate our finding, indicating that SUV has little prognostic value in patients with DLBCL. ${ }^{9,13,29,30,34}$ Prognosis appears to be driven by metabolic tumor burden and maximum FDG avidity; although two patients may have widely different tumor burdens, they can have a similar SUV $_{\max }$ for the entire body, as their most active lesions are equally FDG-avid.

One more important finding that should be highlighted is the association between TMTV and median time to treatment randomization. This finding emphasizes the recognition and need for avoidance of any potential delays in initiation of treatment, particularly in patients with a high tumor burden.

Greater optimization and standardization of quantitative PET parameters and methodology would markedly improve their utilization in clinical practice. Furthermore, automation of TMTV assessment using image processing could enable rapid availability of reproducible results following scans. ${ }^{26,35,36}$ Other factors requiring further investigation include identification of patient subpopulations who would benefit most from applying PET-based tumor metrics; combination of PET-based tumor metrics with other predictive or prognostic tools (e.g., gene expression profiling, circulating tumor DNA, end-of-treatment PET or minimal residual disease); the role of TMTV/TLG in personalization of treatment; and the use of more specific PET radiotracers. Currently, assessment of TMTV alone or in combination with other risk factors is not regularly used in clinical practice. Such assessments may become more routine with the growing interest in individualized treatment strategies driven by the increasing availability of targeted treatments.

The current analysis is limited by the fact that it was post hoc, rather than a prospectively defined analysis. However, the prospective nature of the trial with standardized PET procedures and the large sample size of PET-CT scans available at baseline provide confidence in the conclusions drawn from the data. Although the treatment arms from GOYA were combined, there is no evidence of a difference in PFS or OS between obinutuzumab-CHOP and rituximab-CHOP after long-term follow up of the study. ${ }^{37}$

In conclusion, these data from the phase III GOYA study further demonstrate the prognostic value of baseline quantitative PET metrics, as well as their incremental prognostic value in combination with IPI score or cell-oforigin classification, for predicting survival in a previously untreated DLBCL patient population receiving immunochemotherapy. The identification of patients with the worst prognosis prior to initiation of first-line treatment may lead to timely changes, from planned therapy to alternative treatment strategies, tailored to provide greater clinical benefit to high-risk patients. However, integration of PET-derived metrics with molecular and clinical data will likely be necessary to exclude confounding variables and improve their prognostic/predictive value in the future.

\section{Disclosures}

LK reports consultancy fees from F. Hoffmann-La Roche $L t d$, Genentech, Inc. and travel expenses from F. HoffmannLa Roche Ltd; FM is an employee of F. Hoffmann-La Roche Ltd; MM reports consulting, advisory board role and speaker's bureau for F. Hoffmann-La Roche Ltd, Janssen, Novartis, Gilead Sciences and Sandoz; and travel expenses from F. Hoffmann-La Roche Ltd; LS reports research funding from Teva; and consultancy and honoraria fees from $F$. Hoffmann-La Roche, Genentech, Inc., AbbVie, Amgen, Apobiologix, Acerta, AstraZeneca, Celgene, Debiopharm, Gilead, Incyte, Janssen, Kite Pharma, Karyopharm, Lundbeck, Merck, MorphoSys, Novartis, Seattle Genetics, Sandoz, Takeda, Teva, TG Therapeutics and Verastem; DB reports research funding from and consulting or advisory role for F. Hoffmann-La Roche Ltd, Gilead Sciences, Janssen-Cilag, Seattle Genetics, Morphosys; NC reports research funding 
from F. Hoffmann-La Roche Ltd and honoraria, consultancy and advisory board fees from F. Hoffmann-La Roche Ltd, Merck, Bristol-Meyers Squibb, AstraZeneca, Amgen and Lundbeck; $E G B$ reports consultancy or advisory role for AbbVie, Celgene, EUSA Pharma, Gilead Sciences, Janssen and Koiwa; speakers' bureau from AbbVie, Janssen and Takeda; and travel expenses from AbbVie, Janssen and $F$. Hoffmann-La Roche Ltd.; AP reports honoraria from and speaker's bureau for Bristol-Myers Squibb, F. Hoffmann-La Roche Ltd; Janssen, Celgene, Gilead Sciences and Mundipharma EDO; consultancy or advisory fees from BristolMyers Squibb, F. Hoffmann-La Roche Ltd, Celgene, Gilead Sciences, Mundipharma EDO; patents, royalties, other intellectual property from Mundipharma EDO and travel expenses from F. Hoffmann-La Roche Ltd. CG; XH; YS and YT declare no conflicts of interest; $C B$ is an employee of Genentech, Inc. and has equity ownership in F. Hoffmann-La Roche Ltd; AK is an employee of F. Hoffmann-La Roche Ltd; GS is an employee and stockholder in F. Hoffmann-La Roche Ltd and reports travel expenses from F. HoffmannLa Roche Ltd.; TN and DS are employees and stockholders of F. Hoffmann-La Roche Ltd; UV reports consulting or advisory role for Janssen, Celgene, Juno Therapeutics, Kite Pharma; speaker's bureau for F. Hoffmann-La Roche Ltd, Janssen, Celgene, Gilead Sciences, Servier, Abbvie; research funding from Celgene; travel expenses from Celgene, $F$. Hoffmann-La Roche Ltd, AbbVie and advisory board session for Genmab; MT reports honoraria and consultancy fees from Janssen, Gilead Sciences, Bristol-Meyers Squibb, Amgen, AbbVie, Takeda, F. Hoffmann-La Roche Ltd and MorphoSys and honoraria from Incyte.

\section{Contributions}

$L K, M M, D S$ and $T N$ designed the study; $L K, M M, T N$ and $D S$ conducted the study; $A P, M M, U V, C B, E G-B, A P, D B, Y T, Y S$,
LS, CG, NC, MT and XH recruited and followed up patients; $T N, G S, D S$ and $A K$ collected data; FM, LK and DS analyzed data; $F M, L K, T N, U V, C B, E-G B, A P, D B, A K, Y T, Y S, L S, C G$, $N C, M T$ and $X H$ interpreted data. All authors were involved in the writing of the manuscript, provided their final approval of the manuscript and are accountable for all aspects of the work.

\section{Acknowledgments}

The authors wish to thank the GOYA study team investigators, coordinators, nurses, and patients and are grateful to Will Harris and Joseph Paulson for providing statistical support for the manuscript.

\section{Funding}

The GOYA study was supported by F. Hoffmann-La Roche $L t d$, with scientific support from the Fondazione Italiana Linfomi. Editorial support (in the form of writing assistance, collating author comments, assembling tables/figures, grammatical editing, and referencing) under the direction of Lale Kostakoglu was provided by Katie Smith, PhD, and Molly Heitz, PhD, of Ashfield MedComms, an Ashfield Health company, and funded by F. Hoffmann-La Roche Ltd.

\section{Data-sharing statement}

Qualified researchers may request access to individual patient level data through the clinical study data request platform (https://vivli.org/). Further details on Roche's criteria for eligible studies are available here (https://vivli.org/members/ourmembers/). For further details on Roche's Global Policy on the Sharing of Clinical Information and how to request access to related clinical study documents, see here (https://www.roche. com/research_and_development/who_we_are_how_we_work/clini cal_trials/our_commitment_to_data_sharing.htm).

\section{References}

1. National Comprehensive Cancer Network (NCCN). NCCN clinical practice guidelines in oncology (NCCN Guidelines): B-cell lymphomas. 2020 Version 4.2020. Available from: https://www.nccn.org/professionals/physician_gls/pdf/bcell.pdf.

2. Tilly H, Gomes da Silva M, Vitolo U, et al. Diffuse large B-cell lymphoma (DLBCL): ESMO clinical practice guidelines for diagnosis, treatment and follow-up. Ann Oncol. 2015;26(Suppl 5):S116-125.

3. Alizadeh AA, Eisen MB, Davis RE, et al. Distinct types of diffuse large B-cell lymphoma identified by gene expression profiling. Nature. 2000;403(6769):503-11.

4. Chapuy B, Stewart C, Dunford AJ, et al. Molecular subtypes of diffuse large B cell lymphoma are associated with distinct pathogenic mechanisms and outcomes. Nat Med. 2018;24(5):679-690.

5. Chaganti $S$, Illidge $T$, Barrington $S$, et al. Guidelines for the management of diffuse large B-cell lymphoma. $\mathrm{Br} J$ Haematol.
2016;174(1):43-56.

6. Sehn LH, Berry B, Chhanabhai M, et al. The Revised International Prognostic Index (R-IPI) is a better predictor of outcome than the standard IPI for patients with diffuse large B-cell lymphoma treated with R-CHOP. Blood. 2007;109(5):1857-1861.

7. El-Galaly TC, Villa D, Alzahrani M, et al. Outcome prediction by extranodal involvement, IPI, R-IPI, and NCCN-IPI in the PET/CT and rituximab era: a Danish-Canadian study of 443 patients with diffuse-large B-cell lymphoma. Am J Hematol. 2015;90(11):1041-1046.

8. Kim TM, Paeng JC, Chun IK, et al. Total lesion glycolysis in positron emission tomography is a better predictor of outcome than the International Prognostic Index for patients with diffuse large B cell lymphoma. Cancer. 2013;119(6):1195-1202.

9. Mikhaeel NG, Smith D, Dunn JT, et al. Combination of baseline metabolic tumour volume and early response on PET/CT improves progression-free survival prediction in DLBCL. Eur $\mathrm{J}$ Nucl Med Mol Imaging. 2016;43(7):1209-1219. 
10. Song MK, Yang DH, Lee GW, et al. High total metabolic tumor volume in $\mathrm{PET} / \mathrm{CT}$ predicts worse prognosis in diffuse large $\mathrm{B}$ cell lymphoma patients with bone marrow involvement in rituximab era. Leuk Res. 2016;42:1-6.

11. Sasanelli M, Meignan M, Haioun C, et al. Pretherapy metabolic tumour volume is an independent predictor of outcome in patients with diffuse large B-cell lymphoma. Eur J Nucl Med Mol Imaging. 2014;41(11):2017-2022.

12. Cottereau AS, Lanic $H$, Mareschal $S$, et al. Molecular profile and FDG-PET/CT total metabolic tumor volume improve risk classification at diagnosis for patients with diffuse large B-cell lymphoma. Clin Cancer Res. 2016;22(15):3801-3809.

13. Toledano MN, Desbordes P, Banjar A, et al. Combination of baseline FDG PET/CT total metabolic tumour volume and gene expression profile have a robust predictive value in patients with diffuse large B-cell lymphoma. Eur J Nucl Med Mol Imaging. 2018;45(5):680-688.

14. Chang CC, Cho SF, Chuang YW, et al. Prognostic significance of total metabolic tumor volume on (18)F-fluorodeoxyglucose positron emission tomography/ computed tomography in patients with diffuse large B-cell lymphoma receiving rituximab-containing chemotherapy. Oncotarget. 2017;8(59):99587-99600.

15. Vercellino L, Cottereau A-S, Casasnovas O, et al. High total metabolic tumor volume at baseline predicts survival independent of response to therapy. Blood. 2020;135(16): 1396-1405.

16. Mamot $C$, Klingbiel D, Hitz F, et al. Final results of a prospective evaluation of the predictive value of interim positron emission tomography in patients with diffuse large B-cell lymphoma treated with R-CHOP-14 (SAKK 38/07). J Clin Oncol. 2015;33(23):2523-2529.

17. Pregno $P$, Chiappella $A$, Bellò $M$, et al. Interim 18-FDG-PET/CT failed to predict the outcome in diffuse large $\mathrm{B}$-cell lymphoma patients treated at the diagnosis with rituximab-CHOP. Blood. 2012;119(9):2066-2073.

18. Bishton MJ, Hughes S, Richardson F, et al. Delineating outcomes of patients with diffuse large $b$ cell lymphoma using the national comprehensive cancer network-international prognostic index and positron emission tomography-defined remission status; a population-based analysis. $\mathrm{Br} \mathrm{J}$ Haematol. 2016;172(2):246-254.

19. Kanemasa Y, Shimoyama T, Sasaki Y, et al. Analysis of prognostic value of complete response by PET-CT and further stratification by clinical and biological markers in DLBCL patients. Med Oncol. 2017;34(2):29.

20. Kostakoglu L, Martelli M, Sehn LH, et al. End-of-treatment PET/CT predicts PFS and overall survival in DLBCL after firstline treatment: results from GOYA. Blood Adv. 2021;5(5):1283-1290.

21. Vitolo $U$, Trněný $M$, Belada $D$, et al. Obinutuzumab or rituximab plus cyclophosphamide, doxorubicin, vincristine, and prednisone in previously untreated diffuse large B-cell lymphoma. J Clin Oncol. 2017;35(31):3529-3537.

22. Marcus R, Davies A, Ando K, et al. Obinutuzumab for the firstline treatment of follicular lymphoma. N Engl J Med. 2017;377(14):1331-1344.

23. Goede V, Fischer K, Busch R, et al. Obinutuzumab plus chlorambucil in patients with CLL and coexisting conditions. $\mathrm{N}$ Engl J Med. 2014;370(12):1101-1110.
24. Shagera QA, Cheon GJ, Koh Y, et al. Prognostic value of metabolic tumour volume on baseline (18)F-FDG PET/CT in addition to NCCN-IPI in patients with diffuse large B-cell lymphoma: further stratification of the group with a high-risk NCCN-IPI. Eur J Nucl Med Mol Imaging. 2019;46(7):1417-1427.

25. Gormsen LC, Vendelbo MH, Pedersen MA, et al. A comparative study of standardized quantitative and visual assessment for predicting tumor volume and outcome in newly diagnosed diffuse large B-cell lymphoma staged with 18F-FDG PET/CT. Eur J Nucl Med Mol Imaging Research. 2019;9(1):36.

26. Ilyas H, Mikhaeel NG, Dunn JT, et al. Defining the optimal method for measuring baseline metabolic tumour volume in diffuse large B cell lymphoma. Eur J Nucl Med Mol Imaging. 2018;45(7):1142-1154.

27. Xie M, Zhai W, Cheng S, Zhang $H$, Xie Y, He W. Predictive value of F-18 FDG PET/CT quantization parameters for progression-free survival in patients with diffuse large B-cell lymphoma. Hematology. 2016;21(2):99-105.

28. Xie M, Wu K, Liu Y, Jiang Q, Xie Y. Predictive value of F-18 FDG $\mathrm{PET} / \mathrm{CT}$ quantization parameters in diffuse large $\mathrm{B}$ cell lymphoma: a meta-analysis with 702 participants. Med Oncol. 2015;32(1):446.

29 Ceriani L, Milan L, Martelli M, et al. Metabolic heterogeneity on baseline 18FDG-PET/CT scan is a predictor of outcome in primary mediastinal B-cell lymphoma. Blood. 2018;132(2):179-186.

30. Delaby G, Hubaut MA, Morschhauser F, et al. Prognostic value of the metabolic bulk volume in patients with diffuse large B-cell lymphoma on baseline (18)F-FDG PET-CT. Leuk Lymphoma. 2020;61(7):1584-1591.

31. Guo B, Tan X, Ke Q, Cen H. Prognostic value of baseline metabolic tumor volume and total lesion glycolysis in patients with lymphoma: a meta-analysis. PLoS One. 2019;14(1):e0210224.

32. Esfahani SA, Heidari P, Halpern EF, Hochberg EP, Palmer EL, Mahmood U. Baseline total lesion glycolysis measured with (18)F-FDG PET/CT as a predictor of progression-free survival in diffuse large B-cell lymphoma: a pilot study. Am J Nucl Med Mol Imaging. 2013;3(3):272-281.

33. Zhou M, Chen Y, Huang H, Zhou X, Liu J, Huang G. Prognostic value of total lesion glycolysis of baseline $18 \mathrm{~F}-$ fluorodeoxyglucose positron emission tomography/computed tomography in diffuse large B-cell lymphoma. Oncotarget. 2016;7(50):83544-83553.

34. Zhang YY, Song L, Zhao MX, Hu K. A better prediction of progression-free survival in diffuse large B-cell lymphoma by a prognostic model consisting of baseline TLG and $\% \Delta S U V_{\max }$. Cancer Med. 2019;8(11):5137-5147.

35. Jemaa S, Fredriskson J, Coimbra Aea. A fully automated measurement of total metabolic tumor burden in diffuse large B-cell lymphoma and follicular lymphoma. Blood. 2019;134(Suppl 1):S4666.

36. Burggraaff CN, Rahman F, Kaßner I, et al. Optimizing workflows for fast and reliable metabolic tumor volume measurements in diffuse large C-cell lymphoma. Mol Imaging Biol. 2020;22(4):1102-1110.

37. Sehn LH, Martelli M, Trnenný M, et al. Final analysis of GOYA: a randomized, open-label, Phase III study of obinutuzumab or rituximab plus $\mathrm{CHOP}$ in patients with previously untreated diffuse large B-cell lymphoma. Blood. 2019;134(Suppl 1):S4088. 